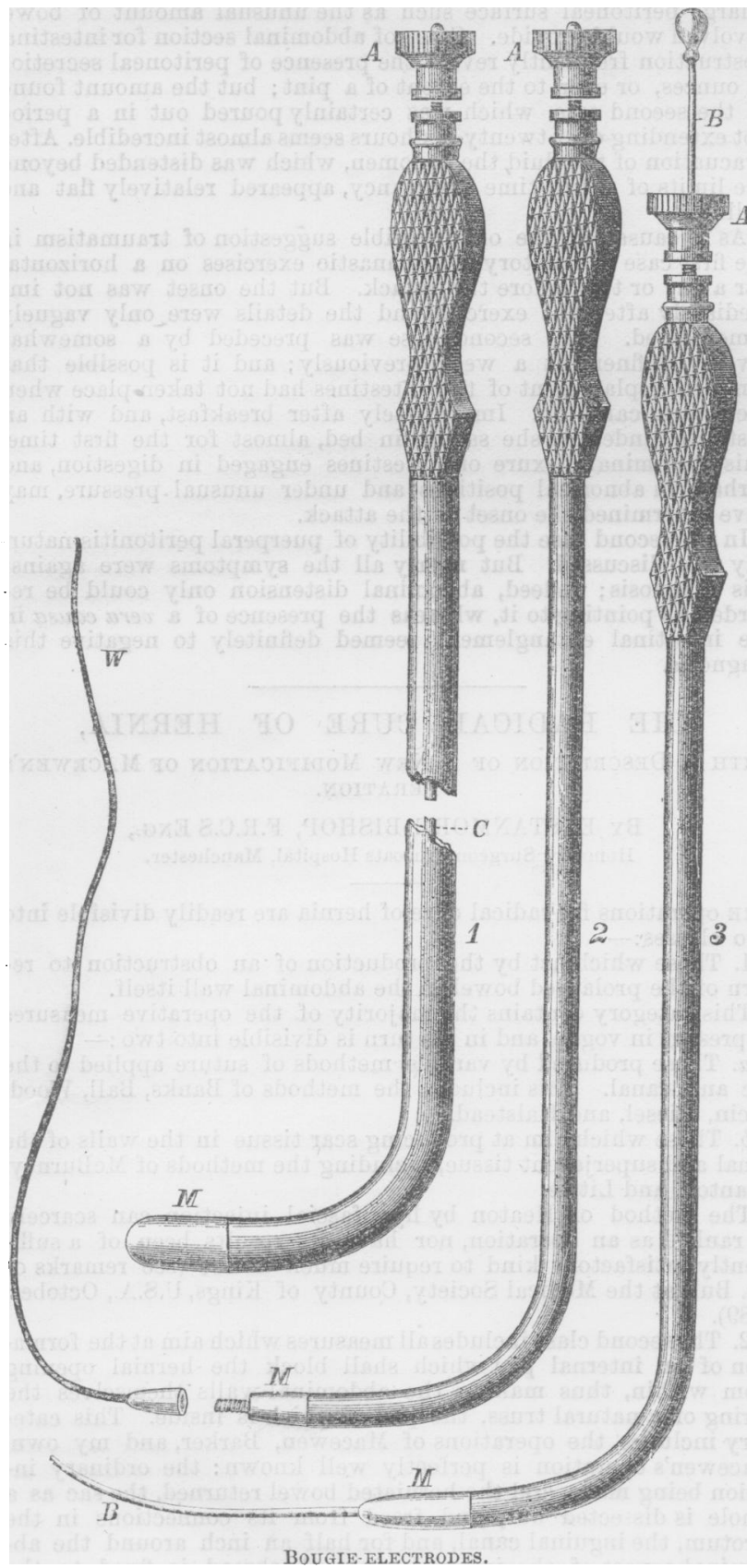

For most purposes, a series of bougies of No. 1 pattern will be sufficient, and the small sizes are not so essential as the larger, for in some instances I have rapidly dilated the stricture first to No. 5 or 6 English, and then used electrolysis subsequently. The bougies can be made stiff and unyielding or thoroughly flexible so that they can be bent into any shape the operator may prefer. Number 2 instrument is a most useful form, but it has this disadvantage, that the current acts to a slight extent on the whip bougie $(W)$ where it is screwed into the metal, and if it is not carefully examined from time to time, there is no doubt some slight risk of its being left behind in the bladder. At one $\operatorname{tim} \theta \mathrm{I}$ deemed this objection to be a more serious one than it really is. I am, however, confident, from a somewhat extensive use of this form of instrument, that wiih the most ordinary care this acc:dent is impossible.

(To be continued.)
TWO CASES OF ACUTE INTESTINAL OBSTRUCTION, ASSOCIATED WITH ENORMOUS SECRETION OF PERITONEAL FLUID:

\section{ABDOMINAL SECTION AND} RECOVERY IN EACH.

By J. GREIG SMITH, M.A., F.R.S.E., Surgeon to the Bristol Royal Infirmary; Joint Lecturer on Surgery, Bristol Medical School.

CASE I.-D. D., aged 14, was seen, In consultation with Mr. Firmin Cuthbert and Dr. Edis, at Gloucester on April 18th, 1889 , and operated on on that day.

The following notes were kindly supplied by Mr. Firmin Cuthbert: "With the exception of sluggish bowels, the lad had been quite well until April 11th at 10 A.M., when he was seized with pain in the epigastrium, which lasted till 3 P.M., when he was sick and had relief from pain. At 5 P.M. the pain came on again, and lasted till 8 P.M. He went to bed at 8 P.M., and, at 10 the pain returned and lasted all night, increasing in severity. When I saw him for the first time, at 9 A.M. on April 12th, he was in such great pain that I gave him a hypodermic injection of onesixth of a grain of morphine. This relieved him slightly, but, in about three hours, a similar dose had to be administered. During the previous night he had had a dose of salts and an enema to move his bowels (without effect); I therefore gave him belladonna and opium in pill (quarter grain of each) every two hours.

"On examining the abdomen, I could feel nothing abnormal. The sickness ceased, but the general aspect of the patient and the continued pain indicated acute obstruction from some cause or other. No blood was passed per anum. An enema, administered by myself, brought away some fæces, but increased the pain. During the night he had no pain, and slept well. His tongue in the morning was coated, and there had been no action of the bowels. In the afternoon the pain returned; another injection of morphine (one-sixth of a grain) was given, an enema administered, and the abdominal parietes were painted with a mixtnre of glycerine and belladonna. On the 14th the pain was less; on the 15th it was worse, and his condition was so serious that operation was proposed, but rejected. On the 16 th and 17 th the boy got steadily worse, the sickness increasing and abdominal distension becoming more marked. On the 18th I telegraphed to Mr. Greig Smith with a view to the performance of abdominal section."

The lad when I saw him had the well-known appearance and symptoms of a patient entering the later stages of acute intestinal obstruction. It was evident enough that, if unrelieved, he could scarcely live over another day. The abdomen was greatly distended, the parietes being tense and brawny. There was dulness in both flanks and in the bypogastrium up to the level of the pubes. Examination of the rectum and abdomen revealed nothing.

It was clear that the patient would not bear a prolonged operation; in fact, the administration of an anæsthetic was not free from risk. An incision large enough to admit two fingers was made at the upper level of the dull area just below the umbilicus, and large quantities of clear serous fluid ran out-perhaps about three or four pints in all. The lower abdomen was almost free of intestine, but high up under the diaphragm the intestines were crowded together in an apparently inextricable mass. A little manipulation with the two fingers released something, and coils of bowel at once fell downwards towards the pelvis. I could now feel an agglomerated lump composed of intestinal coils comparatively empty, which I unravelled, pulled down, and spread out in the lower abdomen. Gas could be felt rushing into the liberated coils; and, being satisfied that the cause of the obstruction was overcome, I did nothing more, but at once inserted a drainage tube and closed the wound. A rounded, hard nodule felt in the mesentery was pulled to the surface and examined; it looked like tubercle, but almost certainly was not.

The after history of the case, as reported by Mr. Firm in Cuthbert, who treated him, is devoid of interest. Pain and sickness passed off ; the bowels acted the same night, and with regularity every day following with a few exceptions, on which small enemata were employed. The tube was removed after four days, very little fluid having come away through it-not more than an cunce in all. The temperature was normal throughout the pro- 
gress of the case both before and after operation. He is now (March, 1890) in perfect health.

CASE II.-Mrs. A. F. H., aged 30, was seen in consultation with Mr. Alford, of Weston-super-Mare, at noon, on December 24th, 1889 , and operated on at once.

The history given by Mr. Alford was as follows: On the evening of December 15th the patient had been confined of her first child, after a somewhat tedious labour, by the aid of the long forceps. The after progress was perfectly natural and satisfactory. On the third day the bowels acted well after a dose of castor oil and an enema. There was some little trouble from hæmorrhoids. On the eighth day (December 22nd) the bowels were again thoroughly moved by castor oil and enema. On December 23 rd she had a good night as usual, felt very well, and enjoyed a good breakfast. After breakfast she sat up in bed and was selecting Christmas cards, when she suddenly screamed out with acute pain from "cramp in the bowels," as she said. The pain continued all day, and was of a very violent character, in spite of poultices, turpentine fomentations, anodyne applications, and nepenthe by the mouth. Castor oil was given by the mouth, and large injections of oil and water by the rectum. The abdomen rapidly became distended with flatus. There was no special tenderness on pressure over the abdomen. Towards night dulness was made out in both flanks.

On December 24 th, at 8.30 A.M., she was decidedly worse. She had not slept all night, and the pains had been very severe. Pulse 140, weak and thready ; face drawn and anxious; mouth dry and parched; tongue densely coated with dark fur. No fæces or flatus passed. Abdomen enormously distended, tympanitic in front but dull in the flanks, and for some way upwards in the hypogastrium. Mr. Alford telegraphed to me to come to Weston-superMare prepared to operate for intestinal obstruction.

The patient's condition when I saw her was almost hopeless; it appeared as if she had only a few hours to live. The chance of her living through any operative proceeding, even the slightest, seemed to be almost infinitesimal ; but, after a rapid consultation with Mr. Alford and the patient's husband, it was decided to give her the benefit of this chance. Dr. Rossiter most cautiously and skilfully administered chloroform, and the abdomen was rapidly opened by a short incision a little below the umbilicus.

Clear, straw-coloured fluid at once spouted through the opening to the height of at least a foot, and poured out more quickly than we could collect it, saturating sponges, cloths, sheets, and carpet, and partly filling several vessels. As the main object was rapidity, the fingers were inserted while the fluid continued to flow and the condition inside the abdomen ascertained. The bowels were crowded up under the diaphragm, and under the liver a knot of entangled bowels was definitely and easily discovered. The coils were unravelled and pulled down to the lower abdomen, when they at once became filled with the gas which distended almost to bursting the contiguous intestines. A large glass drainage tube was passed into Douglas's pouch, no attempt being made either to empty the abdomen of fluid or to irrigate it. The whole proceeding occupied only a few minutes, and the patient bore the operation better than was expected.

She was at once put under the most vigorous stimulating treatment, and slowly rallied. During the night she was very ill, but in the morning she had picked up a little strength, and thenceforward she rapidly got well. Forty-two hours after operation there was a free natural action of the bowels. At the end of a week the tube was removed, very little fluid having come away after the first day, when it consisted of fluid unremoved during operation. The patient continues quite well.

REMARKs.- These two cases are recorded together because they seem to have much in common, and because, in respect of some of their features, they are, in my experience, unique. One striking feature was the extraordinary amount of peritoneal effusion, in one case measured by pints, in the other certainly several quarts. Another noteworthy feature was the entanglement of coil upon coil of bowel without any tangible cause such as usually causes obstruction. It seemed in each case as if one half of the intestines were overdistended with gas and free, and the other half twisted and entangled into a confused mass into which gas could not enter. No refined diagnosis was attempted, as it was of great importance in each case to waste no time over any step which was not absolutely essential to the immediate saving of life.

As to the amount of peritoneal secretion present, it could not be accounted for on any other ground than traumatic irritation to a large peritoneal surface such as the unusual amount of bowel involved would provide. Cases of abdominal section for intestinal obstruction frequently reveal the presence of peritoneal secretion in ounces, or even to the extent of a pint; but the amount found in the second case, which was certainly poured out in a period not extending over twenty-six hours seems almost incredible. After evacuation of the fluid the abdomen, which was distended beyond the limits of a full-time pregnancy, appeared relatively flat and hollow.

As to causation, the only possible suggestion of traumatism in the first case is a history of gymnastic exercises on a horizontal bar a day or two before the attack. But the onset was not immediately after the exercise, and the details were only vaguely remembered. The second case was preceded by a somewhat severe confinement a week previously; and it is possible that complete replacement of the intestines had not taken place when the attack came on. Immediately after breakfast, and with an obstetric binder on, she sat up in bed, almost for the first time. This abdominal flexure on intestines engaged in digestion, and perhaps in abnormal positions and under unusual pressure, may have determined the onset of the attack.

In the second case the possibility of puerperal peritonitis naturally was discussed. But nearly all the symptoms were against this diagnosis; indeed, abdominal distension only could be regarded as pointing to it, whereas the presence of a vera causa in the intestinal entanglement seemed definitely to negative this diagnosis.

\section{THE RADICAL CURE OF HERNIA,}

With a Description of a New Modification of Macewen's OPERATION.

BY E. STANMORE BISHOP, F.R.C.S ENG.,

IIonorary Surgeon, Ancoats Hospital, Manchester.

THE operations for radical cure of hernia are readily divisible into two classes:-

1. Those which act by the production of an obstruction to return of the prolapsed bowel in the abdominal wall itself.

This category contains the majority of the operative measures at present in vogue, and in its turn is divisible into two :-

$a$. Those produced by various methods of suture applied to the sac and canal. This includes the methods of Banks, Ball, Wood, Socin, Riesel, and Halstead.

$b$. Those which aim at producing scar tissue in the walls of the canal and superjacent tissue, including the methods of McBurney, Spanton, and Little.

The method of Heaton by hypofascial injection can scarcely be ranked as an operation, nor have its results been of a sufficiently satisfactory kind to require much notice (see remarks of Dr. Bull at the Medical Society, County of Kings, U.S.A., October, 1889).

2. The second class includes all measures which aim at the formation of an internal pad which shall block the hernial opening from within, thus making the abdominal walls themselves the spring of a natural truss, the pad of which is inside. This category includes the operations of Macewen, Barker, and my own. Macewen's operation is perfectly well known: the ordinary incision being made, and the herniated bowel returned, the sac as a whole is dissected out, and freed from its connections in the scrotum, the inguinal canal, and for half an inch around the abdominal aspect of the internal ring. A thread is fixed to the fundus, and carried through and through the sac from side to side up to the external ring; this thread is then passed on through the canal to the abdominal aspect of the fascia transversalis, and there penetrates the abdominal wall about one inch above the internal ring; by passing the thread through and through the muscle, after pulling upon it so as to invaginate the plug so formed, it is fixed, and the sac then lies inside the abdominal opening between the transversalis fascia and the outer surface of the peritoneum, in the space formed for it by freeing the peritoneum from the internal wall. The ring and canal are then closed by a suture so placed that it draws the conjoined tendon in front of Poupart's ligament, and holds it there until consolidation has been effected.

Consideration and practice of this method reveal certain things. It will be noticed:-

1. That the sac must depend after operation for its vitality upon 